\title{
Preliminary evidence for brain complications in obese adolescents with type 2 diabetes mellitus
}

\author{
P. L. Yau • D. C. Javier • C. M. Ryan • W. H. Tsui • \\ B. A. Ardekani $\cdot$ S. Ten $\cdot$ A. Convit
}

Received: 23 March 2010 / Accepted: 24 June 2010 /Published online: 30 July 2010

(C) Springer-Verlag 2010

\begin{abstract}
Aims/hypothesis Central nervous system abnormalities, including cognitive and brain impairments, have been documented in adults with type 2 diabetes who also have multiple co-morbid disorders that could contribute to these observations. Assessing adolescents with type 2 diabetes will allow the evaluation of whether diabetes per se may adversely affect brain function and structure years before clinically significant vascular disease develops.

Methods Eighteen obese adolescents with type 2 diabetes and 18 obese controls without evidence of marked insulin
\end{abstract}

P. L. Yau - D. C. Javier · W. H. Tsui • B. A. Ardekani - A. Convit Department of Psychiatry,

New York University School of Medicine,

New York, NY, USA

\section{M. Ryan}

Department of Psychiatry, University of Pittsburgh,

Pittsburgh, PA, USA

W. H. Tsui $\cdot$ B. A. Ardekani $\cdot$ A. Convit

Nathan Kline Institute for Psychiatric Research,

Orangeburg, NY, USA

\section{S. Ten}

Department of Pediatric Endocrinology,

Maimonides Medical Center,

Brooklyn, NY, USA

\section{A. Convit}

Department of Medicine,

New York University School of Medicine,

New York, NY, USA

\section{A. Convit $(\square)$}

The BODyLab, New York University School of Medicine,

145 32nd Street, 8th Floor,

New York, NY 10016, USA

e-mail: antonio.convit@med.nyu.edu resistance, matched on age, sex, school grade, ethnicity, socioeconomic status, body mass index and waist circumference, completed MRI and neuropsychological evaluations. Results Adolescents with type 2 diabetes performed consistently worse in all cognitive domains assessed, with the difference reaching statistical significance for estimated intellectual functioning, verbal memory and psychomotor efficiency. There were statistical trends for executive function, reading and spelling. MRI-based automated brain structural analyses revealed both reduced white matter volume and enlarged cerebrospinal fluid space in the whole brain and the frontal lobe in particular, but there was no obvious grey matter volume reduction. In addition, assessments using diffusion tensor imaging revealed reduced white and grey matter microstructural integrity.

Conclusions/interpretation This is the first report documenting possible brain abnormalities among obese adolescents with type 2 diabetes relative to obese adolescent controls. These abnormalities are not likely to result from education or socioeconomic bias and may result from a combination of subtle vascular changes, glucose and lipid metabolism abnormalities and subtle differences in adiposity in the absence of clinically significant vascular disease. Future efforts are needed to elucidate the underlying pathophysiological mechanisms.

Keywords Adolescence - Apparent diffusion coefficient . Brain complications Cognitive performance . Diffusion tensor imaging - Fractional anisotropy Obesity - Type 2 diabetes

$\begin{array}{ll}\text { Abbreviations } \\ \text { ADC } & \text { Apparent diffusion coefficient } \\ \text { COWAT } & \text { Controlled Oral Word Association Test } \\ \text { CRP } & \text { C-reactive protein }\end{array}$




$\begin{array}{ll}\text { CSF } & \text { Cerebrospinal fluid } \\ \text { DSST } & \text { Digit Symbol Substitution Test } \\ \text { DTI } & \text { Diffusion tensor imaging } \\ \text { FA } & \text { Fractional anisotropy } \\ \text { FDR } & \text { False discovery rate } \\ \text { FLAIR } & \text { Fast fluid-attenuated inversion recovery } \\ \text { FOV } & \text { Field of view } \\ \text { GM } & \text { Grey matter } \\ \text { HOMA-IR } & \text { HOMA-insulin resistance } \\ \text { MPRAGE } & \text { Magnetisation-prepared rapid acquisition } \\ & \text { gradient echo } \\ \text { MRI } & \text { Magnetic resonance imaging } \\ \text { NEX } & \text { Number of averages } \\ \text { SES } & \text { Socioeconomic status } \\ \text { SPM } & \text { Statistical parametric mapping } \\ \text { SPM2 } & \text { Statistical parametric mapping software } \\ & \text { version 2 } \\ \text { T1 } & \text { Spin-lattice relaxation time } \\ \text { T2 } & \text { Spin-spin relaxation time } \\ \text { TE } & \text { Echo time } \\ \text { TI } & \text { Inversion time } \\ \text { TR } & \text { Repetition time } \\ \text { VANCOVA } & \text { Voxelwise analysis of covariance } \\ \text { WASI } & \text { Wechsler Abbreviated Scale of Intelligence } \\ \text { WCST } & \text { Wisconsin Card Sorting Test } \\ \text { WM } & \text { White matter } \\ \text { WRAML } & \text { Wide Range Assessment of Memory and } \\ & \text { Learning } \\ \text { WRAT } & \text { Wide Range Achievement Test }\end{array}$

\section{Introduction}

Obesity is a major risk factor for the development of type 2 diabetes in adults and children. With the growing prevalence of childhood obesity, the incidence of type 2 diabetes in children and adolescents is increasing at an alarming rate [1]. Type 2 diabetes may become the most common form of paediatric diabetes within the next decade. Older adults with type 2 diabetes are at increased risk for developing micro- and macrovascular complications (e.g. retinopathy, neuropathy, nephropathy and cardiovascular disease) [2] They also show significant cognitive slowing as well as learning and memory deficits [3, 4]. Structural brain abnormalities are also commonly associated with type 2 diabetes among older adults [5-7] but for the most part, those abnormalities tend to reflect non-specific pathology such as increased atrophy, overt white matter pathology or stroke $[3,8]$, and their magnitude tends to be modest. However, more recent studies have demonstrated significantly reduced hippocampal volumes among middle-aged and elderly individuals with type 2 diabetes $[9,10]$.
There have been only a few brain studies in children and young adults with type 1 diabetes, and the findings are so far inconsistent, with one study showing reduced brain volume relative to matched non-diabetic controls [11] but another showing no differences [12]. Young people with type 2 diabetes, despite shorter disease duration and better glucose control, have higher rates of complications (microalbuminuria and hypertension) than their peers with type 1 diabetes [13]. Therefore, we predict that their brains may also be more affected. However, no studies have evaluated cognition or the brain in children or adolescents with type 2 diabetes. Although adolescents with type 2 diabetes do not yet have clinically significant vascular disease, there is evidence that they may have increased carotid artery intima-media thickness and stiffness [14], which has also been reported among children and adolescents with type 1 diabetes [15].

Microstructural integrity of cerebral white and grey matter (WM and GM) has been associated with intact cognitive performance in both adults and children [16, 17]. Although not anticipating finding gross WM disease among obese adolescents with type 2 diabetes, we were interested in exploring whether there are WM microstructural alterations that could adversely affect cognitive functioning in these still-developing brains. Consequently, we used sensitive and unbiased magnetic resonance imaging (MRI) techniques, such as diffusion tensor imaging (DTI) and statistical parametric mapping (SPM)-based segmentation and volumetric analysis, to determine the presence of subtle cerebral alterations among our participants.

The goal of this study was to ascertain, for the first time, whether relative to demographically similar non-diabetic obese adolescents with no evidence of marked insulin resistance, obese adolescents with type 2 diabetes have diminished cognitive performance and subtle abnormalities in brain integrity. Given that obesity is one of the major risk factors for sleep apnoea in children [18] and that obstructive sleep apnoea is known to affect both cognition [19] and the brain [20], the groups were also matched on self-ratings of sleep apnoea. We hypothesised that adolescents with type 2 diabetes would show reductions on measures of overall intellectual function, declarative memory, executive function and psychomotor efficiency. In addition, we predicted that they would have diffuse alterations in cerebral WM and GM integrity, particularly in the frontal and medial temporal regions.

\section{Methods}

Participants

We studied 18 obese adolescents with type 2 diabetes consecutively referred by local area paediatric endocrinol- 
ogists. Eighteen obese adolescents without type 2 diabetes or marked insulin resistance comparable in age, sex, school grade, ethnicity, socioeconomic status (SES) (AB Hollingshead, Department of Sociology, Yale University, unpublished data), BMI and waist circumference were recruited as controls among relatives of other study participants or via internet advertisements. This study was approved by the local Internal Review Board. Informed consent was obtained from parents and assent obtained from each child. The data acquisition for this study was completed in 2009.

All participants underwent medical, endocrine, psychiatric, neuropsychological and brain MRI assessments during a comprehensive 6-7 h evaluation completed over two visits. We excluded individuals with significant medical conditions (other than type 2 diabetes, lipid abnormalities, polycystic ovary disease or hypertension). As we do not anticipate that adolescents with type 2 diabetes or obese controls will have sufficient ischaemic microvessel disease to result in extensive WM hyperintensities on MRI, any occurrence of significant WM hyperintensities in this age group is likely to be indicative of a demyelinating condition such as multiple sclerosis, and this clearly warrants exclusion. We therefore excluded individuals with extensive WM pathology. Also excluded were adolescents with: sexual development Tanner stage less than 4; a psychiatric diagnosis such as depression; current use of psychoactive medications; mental retardation; or significant learning disability.

Standard diagnostic criteria for type 2 diabetes were used and all were beta cell antibody negative. Fasting glucose and insulin values were used to compute HOMA-insulin resistance (HOMA-IR) [21], which has been demonstrated to be a reasonable screening measure of insulin resistance relative to the hyperinsulinaemic-euglycaemic clamp assessment [22]. Control participants had a HOMA-IR $\leq 2.50$, which indicated that they were not likely to have marked insulin resistance. Participants were classified as hypertensive if they received anti-hypertensive treatment or had a sitting blood pressure above the National Cholesterol Education Program cut-off (a systolic $\mathrm{BP} \geq 130 \mathrm{mmHg}$ or a diastolic $\mathrm{BP} \geq 85 \mathrm{mmHg}$ ) [23]. The sleep-related behaviour questionnaire consists of 20 items to be answered 'yes', 'no', or 'don't know' [24]. The self-rating of sleep apnoea depends on the proportion of questions answered yes.

Neuropsychological evaluations

The assessment was conducted in a standardised manner in the postprandial state, split over two sessions each lasting about $1.5 \mathrm{~h}$. We used commonly used normed tests that have been described in detail by Lezak et al. [25]. Overall intellectual functioning was estimated from the Vocabulary and Matrix Reasoning subtests from the Wechsler Abbreviated Scale of Intelligence (WASI). Academic achievement was estimated from the Reading, Spelling and Arithmetic subtests from the Wide Range Achievement Test (WRAT). Verbal, visual and working memory skills were assessed with the Wide Range Assessment of Memory and Learning (WRAML). Attention was measured with the accuracy score from the Digit Vigilance Test and the Attention/Concentration Index Score from the WRAML. Executive function was evaluated with the Wisconsin Card Sorting Test (WCST), the Tower of London Test, and the Controlled Oral Word Association Test (COWAT). Psychomotor efficiency was assessed with the Digit Symbol Substitution Test (DSST). Raw scores are reported, with the exception of the WASI, WRAT and WRAML, which provide aged-corrected standard scores.

MR image acquisition and image analysis

Structural images All participants were studied on the same 1.5 T Siemens Avanto MRI System (Berlin, Germany). Spin-lattice relaxation time (T1)-weighted magnetisation-prepared rapid acquisition gradient echo (MPRAGE; repetition time [TR] $1,300 \mathrm{~ms}$; echo time [TE] $4.38 \mathrm{~ms}$; inversion time [TI] $800 \mathrm{~ms}$; field of view [FOV] $250 \times 250$; slice thickness $1.2 \mathrm{~mm}$; number of averages [NEX] 1 ; flip angle $15^{\circ}$; matrix size $256 \times 256$; 192 coronal slices) and spin-spin relaxation time (T2)weighted (TR 9,000 ms; TE $94 \mathrm{~ms}$; TI 2,000 ms; FOV $210 \times 210$; slice thickness $3 \mathrm{~mm}$; matrix size $256 \times 256$; 50 axial slices) sequences were used as anatomical guides for the DTI analysis. To rule out primary neurological disease and to quantify WM disease, the fast fluid-attenuated inversion recovery (FLAIR; TR 9,000 ms; TE $97 \mathrm{~ms}$; FOV $210 \times 210$; one average and two concatenations; flip angle $145^{\circ}$; slice thickness $3 \mathrm{~mm}$; matrix size $256 \times 256$; 50 axial slices) image was used with the MPRAGE. The modified Fazekas scale [26] was used to rate WM hyperintensities, with scores $>1$ leading to study exclusion. To measure brain microstructural integrity a DTI echo planar sequence (TR 6,100 ms; TE $75 \mathrm{~ms}$; delay in TR=0 ms; b values $0,1,000 \mathrm{~s} / \mathrm{mm}^{2}$; six directions; FOV $210 \times 210$; four averages and one concatenation; slice thickness $3 \mathrm{~mm}$; matrix size $128 \times 128 ; 50$ axial slices) was acquired. To optimise image co-registration, the DTI, T2-weighted and FLAIR images were all obtained in the same axial plane, using the same slice thickness and number.

Diffusion tensor imaging assessment of brain tissue DTI uses the differential water diffusion properties of brain tissues to measure cerebral microstructural integrity and organisation. The more constraint to the movement of 
water, the more anisotropic the diffusion and the more intact the microstructural integrity of the tissue is likely to be. The most commonly used DTI index for assessing WM integrity is fractional anisotropy (FA), ranging in value from 0 (fully isotropic diffusion) to 1 (fully anisotropic diffusion) [27]. Another useful measure is the apparent diffusion coefficient (ADC), which represents the mean magnitude of water diffusivity, thus providing an assessment of tissue density. ADC elevations may represent increased extracellular space, a possible indication of neuronal damage [28], which makes it useful in GM regions.

$W M / G M$ volumetric analysis Spatial normalisation and segmentation of MPRAGE images used automated procedures as described in Good et al. [29] in the statistical parametric mapping software version 2 (SPM2). MPRAGE images were first corrected for signal non-uniformities and spatially normalised to the standard T1 Montreal Neurological Institute template. Using the tissue classification algorithm in SPM2, we segmented the normalised MPRAGE images into their GM, WM and cerebrospinal fluid (CSF) partitions (see Fig. 1), which are maps representing the probability for each voxel being classified as GM, WM and CSF, respectively. These segmented partitions were subsequently normalised to their respective standard templates.

In addition to performing whole-brain assessment, and given that during adolescence frontal lobe myelination is still ongoing [30], we also specifically assessed frontal lobes by using our published reliable frontal lobe parcellation method [31]. We quantified the proportions of WM, GM and CSF volumes in the whole brain and frontal regions at the case level by first mapping the region to each segmented partition and then averaging the values across participants for each of the two groups.

DTI image processing To create the group maps for voxelwise group comparisons a three-step approach was used to correct the images for echo planar acquisition distortions after optimising registration between the T2 and MPRAGE images [32]. The FA and ADC values were computed and maps were generated from the native DTI sequence. Finally, to reduce interpolation errors, all transformations were combined into a single step, which was applied to the FA and ADC maps generated from the native DTI data. Refer to Yau et al. for a detailed description of these methods [33].

\section{Statistical analysis}

We conducted two-tailed independent sample $t$ tests examining group differences in demographics, endocrine
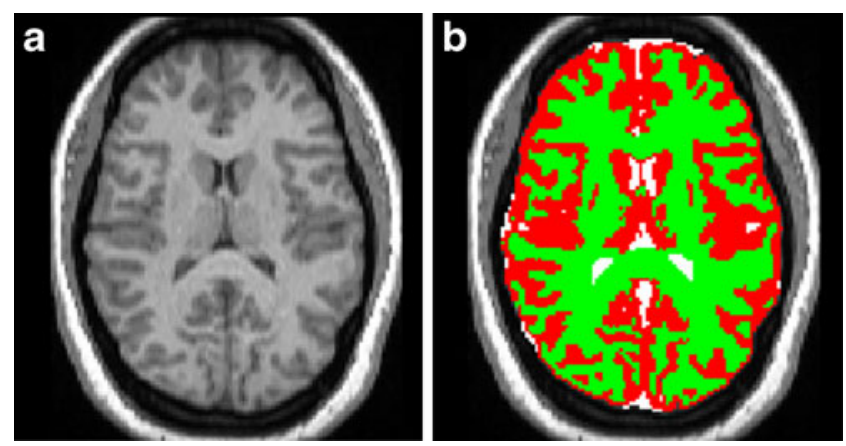

Fig. 1 MPRAGE image segmentation. Individual MPRAGE images are registered to standard space before segmentation is accomplished. a Normalised MPRAGE image. b Segmented image: grey matter (red), white matter (green) and CSF (white) partitions are overlaid on top of the normalised MPRAGE image

data, cognitive data and brain volumes. Variables that were not normally distributed were also tested using the Kolmogorov-Smirnov test. The $\chi^{2}$ test examined group difference in SES. For the imaging data two-tailed voxelwise analysis of covariance (VANCOVA) analyses were performed to examine group differences in whole-brain WM FA and GM ADC, with age as a covariate. WM and GM masks, created from the average MPRAGE image of all participants, were used to confine FA and ADC analyses within those regions. To minimise the chance of type I errors, we restricted the accepted cluster size to those having at least 100 contiguous voxels (each voxel is $1 \mathrm{~mm}^{3}$, thus, the minimum cluster size is $0.1 \mathrm{ml}$ in volume) and by then choosing a false discovery rate (FDR) less than 0.01 . We chose a $p$ value threshold of 0.005 to ensure that the FDR would be kept below 0.01 [34].

\section{Results}

Demographic and endocrine data

There were no group differences in age, sex, school grade, SES, BMI, waist circumference, waist-height ratio or ratings of sleep apnoea. Groups were also comparable in ethnicity. As expected, relative to obese controls, adolescents with type 2 diabetes had significantly higher fasting glucose and insulin levels, HOMA-IR, $\mathrm{HbA}_{1 \mathrm{c}}$ and triacylglycerols, and significantly lower HDL cholesterol levels $(p<0.05$; Table 1$)$. The groups did not differ in total cholesterol, LDL cholesterol, or high-sensitivity C-reactive protein (CRP). Five adolescents with type 2 diabetes and four obese controls had elevated blood pressure, but only one adolescent with diabetes was receiving pharmacological treatment for it. Diabetic participants had been diagnosed with type 2 diabetes for $2.61 \pm$ 1.90 years (range 5 months -7.50 years). The groups did not differ on self-ratings of obstructive sleep apnoea. 
Neuropsychological results

Adolescents with type 2 diabetes consistently scored lower than obese controls on all cognitive tests administered (Table 2). They had significantly lower overall intellectual functioning, lower verbal memory scores, lower psychomotor efficiency (all with $p<0.05$ ), and tended to have more perseverative errors on the WCST $(p=0.06)$. Note that all of these measures showed medium-large to large effect sizes. Despite no group differences in school grade or SES, diabetic participants also performed worse on the WRAT achievement tests, reading $(p=0.09)$ and spelling $(p=0.07)$, both trending toward significance and with medium effect sizes. The three cognitive tests (i.e. the WASI, the Digit Vigilance Test and the WCST) that were not normally distributed in one or both of the groups were also analysed using non-parametric statistics (Kolmogorov-Smirnov) and the results were identical; therefore, for ease of presentation in Table 2 we show only the results from the $t$ tests. Additionally, we confirmed that ratings of sleep apnoea were not associated with any of the cognitive measures that differentiated the groups.

\section{MRI results}

Two control participants had missing MRI scans. Of the 34 participants with MRI scans, only one adolescent with type 2 diabetes had periventricular punctate hyperintensities (rated 1 on the modified Fazekas scale). This very subtle level of abnormality is not considered clinically significant and was not mentioned in this individual's neuroradiological reports.

Volumetric analysis of the segmented partitions revealed that there were no GM volume reductions, but obese adolescents with type 2 diabetes had significantly smaller WM volumes and enlarged CSF space for the whole brain and frontal lobe ( $p \leq 0.005$; all with large effect sizes). These differences remained significant after adjusting for age (Table 3).

DTI VANCOVA revealed both WM and GM microstructural abnormalities among diabetic participants, independent of age (Table 4). When we contrasted the two groups, we found that among obese adolescents with type 2 diabetes there was a total of five clusters of WM FA reductions $(p<0.005)$ with a total volume of 725 voxels or $0.725 \mathrm{ml}$. The type 2 diabetes group only had one small cluster demonstrating significant WM FA elevation. In addition, there were 27 clusters showing GM ADC elevations $(p<0.005)$ among adolescents with type 2 diabetes, 20 of which were in the cerebrum. The cerebral clusters had a total volume of 4,012 voxels or $4.01 \mathrm{ml}$. In exploratory analyses with a less conservative $p$ value threshold of 0.01 , we identified a total of 67 FA or ADC significant clusters, only five of which showed higher FA or lower ADC values in type 2 diabetes.

Given the extensive number of clusters of abnormality, we chose to only itemise in Table 4 the largest clusters using the more conservative $p$ value threshold of less than

Table 1 Demographic and endocrine data

\begin{tabular}{|c|c|c|c|c|c|}
\hline Variable & Type 2 diabetes $(n=18)$ & Controls $(n=18)$ & $t$ & $p$ value & Cohen's $d$ \\
\hline Age (years) & $16.46 \pm 1.89$ & $17.16 \pm 1.45$ & 1.26 & 0.22 & 0.42 \\
\hline Education (years) & $10.75 \pm 1.53$ & $11.15 \pm 1.66$ & 0.77 & 0.45 & 0.26 \\
\hline BMI $\left(\mathrm{kg} / \mathrm{m}^{2}\right)$ & $37.70 \pm 6.36$ & $36.80 \pm 7.22$ & -0.39 & 0.70 & -0.13 \\
\hline SES category ${ }^{\mathrm{a}}$ & $2.94 \pm 1.20$ & $3.43 \pm 1.16$ & $\chi^{2}=2.06$ & 0.26 & \\
\hline $\mathrm{HbA}_{1 \mathrm{c}}(\%)^{\mathrm{b}}$ & $8.32 \pm 2.87$ & $5.29 \pm 0.33$ & -4.32 & $<0.001$ & -1.46 \\
\hline HOMA-IR ${ }^{b}$ & $10.05 \pm 8.00$ & $1.59 \pm 0.65$ & -4.34 & $<0.001$ & -1.47 \\
\hline Fasting glucose $(\mathrm{mmol} / \mathrm{l})^{\mathrm{b}}$ & $8.37 \pm 4.79$ & $4.16 \pm 0.43$ & -3.60 & $<0.01$ & -1.22 \\
\hline Fasting insulin $(\mathrm{pmol} / \mathrm{l})^{\mathrm{b}}$ & $217.45 \pm 189.18$ & $59.94 \pm 23.89$ & -3.41 & 0.001 & -1.15 \\
\hline Triacylglycerol (mmol/l) & $1.42 \pm 0.60$ & $0.95 \pm 0.51$ & -2.48 & 0.02 & -0.85 \\
\hline Total cholesterol $(\mathrm{mmol} / \mathrm{l})^{\mathrm{b}}$ & $4.59 \pm 1.08$ & $4.22 \pm 0.67$ & -1.20 & 0.24 & -0.41 \\
\hline HDL cholesterol (mmol/1) & $0.98 \pm 0.23$ & $1.14 \pm 0.21$ & 2.23 & 0.03 & 0.75 \\
\hline LDL cholesterol $(\mathrm{mmol} / \mathrm{l})$ & $2.82 \pm 0.95$ & $2.65 \pm 0.60$ & -0.63 & 0.53 & -0.22 \\
\hline CRP (nmol/l) & $27.52 \pm 19.33$ & $22.29 \pm 16.38$ & -0.78 & 0.44 & -0.30 \\
\hline Sleep apnoea ratings & $0.23 \pm 0.16$ & $0.17 \pm 0.15$ & -1.01 & 0.32 & -0.38 \\
\hline Waist circumference $(\mathrm{cm})$ & $112.72 \pm 15.36$ & $109.44 \pm 15.99$ & -0.59 & 0.56 & -0.21 \\
\hline Waist-height ratio & $0.67 \pm 0.10$ & $0.64 \pm 0.10$ & -0.78 & 0.44 & -0.28 \\
\hline
\end{tabular}

Data are mean \pm SD

${ }^{\mathbf{a}}$ SES category ranges from 1 to 5 with higher number indicating lower socioeconomic class; ${ }^{\mathbf{b}}$ Adjusted for unequal group variances 
Table 2 Neuropsychological results

\begin{tabular}{|c|c|c|c|c|c|}
\hline Neuropsychological measure & Type 2 diabetes $(n=18)$ & Controls $(n=18)$ & $t$ & $p$ value & Cohen's $d$ \\
\hline \multicolumn{6}{|c|}{ Intellectual capacity and academic achievements } \\
\hline Estimated full-scale IQ & $87.83 \pm 12.55$ & $103.63 \pm 11.75$ & 3.77 & $<0.001$ & 1.30 \\
\hline WRAT reading standard score & $97.18 \pm 15.97$ & $106.44 \pm 14.34$ & 1.75 & 0.09 & 0.61 \\
\hline WRAT spelling standard score & $91.41 \pm 15.88$ & $100.88 \pm 13.42$ & 1.84 & 0.07 & 0.64 \\
\hline WRAT arithmetic standard score & $89.29 \pm 14.92$ & $94.06 \pm 10.52$ & 1.05 & 0.30 & 0.37 \\
\hline \multicolumn{6}{|l|}{ Memory } \\
\hline WRAML verbal memory index & $97.94 \pm 11.59$ & $106.44 \pm 12.09$ & 2.12 & 0.04 & 0.72 \\
\hline WRAML visual index & $101.41 \pm 10.82$ & $102.94 \pm 11.57$ & 0.40 & 0.69 & 0.14 \\
\hline WRAML working memory index & $95.88 \pm 13.91$ & $98.28 \pm 13.86$ & 0.51 & 0.61 & 0.17 \\
\hline \multicolumn{6}{|l|}{ Attention/psychomotor efficiency } \\
\hline DSST & $52.61 \pm 8.04$ & $60.13 \pm 12.64$ & 2.07 & 0.05 & 0.73 \\
\hline Digit vigilance, total omissions & $12.44 \pm 11.06$ & $10.06 \pm 7.12$ & -0.74 & 0.47 & -0.25 \\
\hline WRAML attention/concentration index & $98.53 \pm 17.27$ & $103.39 \pm 14.36$ & 0.91 & 0.37 & 0.31 \\
\hline \multicolumn{6}{|l|}{ Executive functions } \\
\hline WCST perseverative errors ${ }^{\mathrm{a}}$ & $17.47 \pm 13.59$ & $9.47 \pm 6.94$ & -2.03 & 0.06 & -0.74 \\
\hline Tower of London-excess moves & $16.87 \pm 14.18$ & $14.94 \pm 11.89$ & -0.42 & 0.68 & -0.15 \\
\hline COWAT sum of scores & $32.83 \pm 8.05$ & $33.35 \pm 6.81$ & 0.21 & 0.84 & 0.07 \\
\hline
\end{tabular}

Data are mean $\pm \mathrm{SD}$

${ }^{a}$ Adjusted for unequal group variances

0.005. These same sets of selected clusters are displayed on an average brain structural image in Figs $2 \mathrm{a}$ (FA) and $2 \mathrm{~b}$ (ADC).

\section{Discussion}

This is the first report of brain and cognitive abnormalities among obese adolescents with type 2 diabetes. We demonstrate that, in the absence of clinically significant vascular disease, there may be clear brain complications among adolescents with type 2 diabetes. These present predominantly as whole-brain and frontal WM volume reductions and diffuse WM and GM microstructural abnormalities. In addition, we find that relative to matched obese controls, general intellectual ability, verbal memory, executive function, psychomotor efficiency and measures of academic achievement-particularly reading and spelling skills-are lower among adolescents with type 2 diabetes. The intellectual capacity and verbal memory impairments are consistent with those reported among middle-aged adults with well-controlled type 2 diabetes [9], but unlike adults with type 2 diabetes, the reductions in cognitive performance were not restricted to those two domains.

Our DTI analyses demonstrated that, compared with relatively insulin-sensitive obese controls, obese adolescents with type 2 diabetes showed FA reductions in WM tracts in the temporal stem, cingulate, frontal lobe, occipital lobe and cerebral peduncles. During adolescence, the brain continues to undergo the morphological and functional changes associated with continued WM maturation [35], and because of this the observed WM volume and FA reductions may represent delayed WM maturation or axonal damage. In youths with type 1 diabetes, hyperglycaemia has been shown to be associated with smaller grey and white matter volumes despite no volume reduction relative to age-matched controls [12]. Hyperglycaemia-associated
Table 3 Brain volumetric data

Data are mean \pm SD

Volumes in $\mathrm{ml}$

\begin{tabular}{lllrrr}
\hline Variable & Type 2 diabetes $(n=18)$ & Controls $(n=18)$ & $t$ & $p$ value & Cohen's $d$ \\
\hline Whole-brain GM volume & $917.23 \pm 98.30$ & $943.19 \pm 64.20$ & 0.90 & 0.38 & 0.31 \\
Whole-brain WM volume & $511.65 \pm 31.56$ & $543.89 \pm 31.20$ & 2.99 & 0.01 & 1.03 \\
Whole-brain CSF volume & $419.22 \pm 51.51$ & $365.42 \pm 41.84$ & -3.32 & 0.01 & -1.14 \\
Frontal GM volume & $357.99 \pm 121.27$ & $372.20 \pm 75.15$ & 1.15 & 0.26 & 0.40 \\
Frontal WM volume & $232.44 \pm 51.75$ & $251.21 \pm 40.69$ & 3.24 & $<0.01$ & 1.11 \\
Frontal CSF volume & $171.65 \pm 49.00$ & $149.64 \pm 48.75$ & -3.64 & $<0.001$ & -1.25 \\
\hline
\end{tabular}


Table 4 Clusters of FA reductions or $\mathrm{ADC}$ elevations in type 2 diabetes $(p<0.005)$
Voxel size, $1 \mathrm{~mm}^{3}$

* Remained significant at $p<0.001$, two-tailed

\begin{tabular}{llcrrr}
\hline Clusters & Size (voxels) & Mean $t$ value & \multicolumn{2}{c}{ Talairach coordinates } \\
\cline { 4 - 6 } & & & $x$ & $y$ & $z$ \\
\hline FA white matter clusters & & & & & \\
$\quad$ Right cingulate WM & 201 & -3.36 & -14.1 & 52.1 & 17.5 \\
$\quad$ Left cerebral peduncle & 182 & -3.45 & 16.1 & 35.5 & -47.2 \\
$\quad$ Left temporal stem & 130 & -3.36 & 51.3 & 41.5 & -30.6 \\
ADC grey matter clusters & & & & & \\
$\quad$ Right superior temporal gyrus & 526 & $3.43^{*}$ & -48.2 & 16.0 & -26.2 \\
$\quad$ Left prefrontal cortex & 382 & $3.48^{*}$ & 43.1 & -2.0 & 9.5 \\
Right parietal cortex & 184 & $3.58^{*}$ & -52.7 & 69.9 & 22.1 \\
\hline
\end{tabular}

oxidative stress may also induce selective oligodendrocyte death, resulting in demyelination [36]. Although hypertensionrelated ischaemic damage remains a possible mechanism for WM damage [37], our voxelwise DTI results remained largely unchanged even after controlling for hypertension. In the absence of group differences in whole-brain or frontal GM volumes, our findings of diffuse ADC elevations in GM among diabetic individuals suggest reductions in GM density. The exact nature or degree of GM alterations remains to be better characterised with more direct assessments using techniques such as spectroscopy or assessment of tissue properties such as T1 relaxation.

We did not assess whether our participants had significant occlusive vascular disease as there is no clear rationale for anticipating clinically significant vascular abnormalities in adolescents. We propose that the negative impact of type 2 diabetes on the adolescent brain may result from a combination of functional vascular changes and glucose and lipid metabolism abnormalities in the absence of overt vascular disease. Type 2 diabetes is known to lead to an altered vascular physiology, such as reductions in endothelial dependent vasodilatation and deficits in cerebral vascular reactivity to $\mathrm{CO}_{2}$ [38]. During brain activation, such as occurs when performing a cognitive task, there is an increase in synaptic activity in the brain region involved. In the normal brain this results in regional vasodilation and thus an increase in glucose availability to that region to support the increased cognitive demand [39]. Therefore,

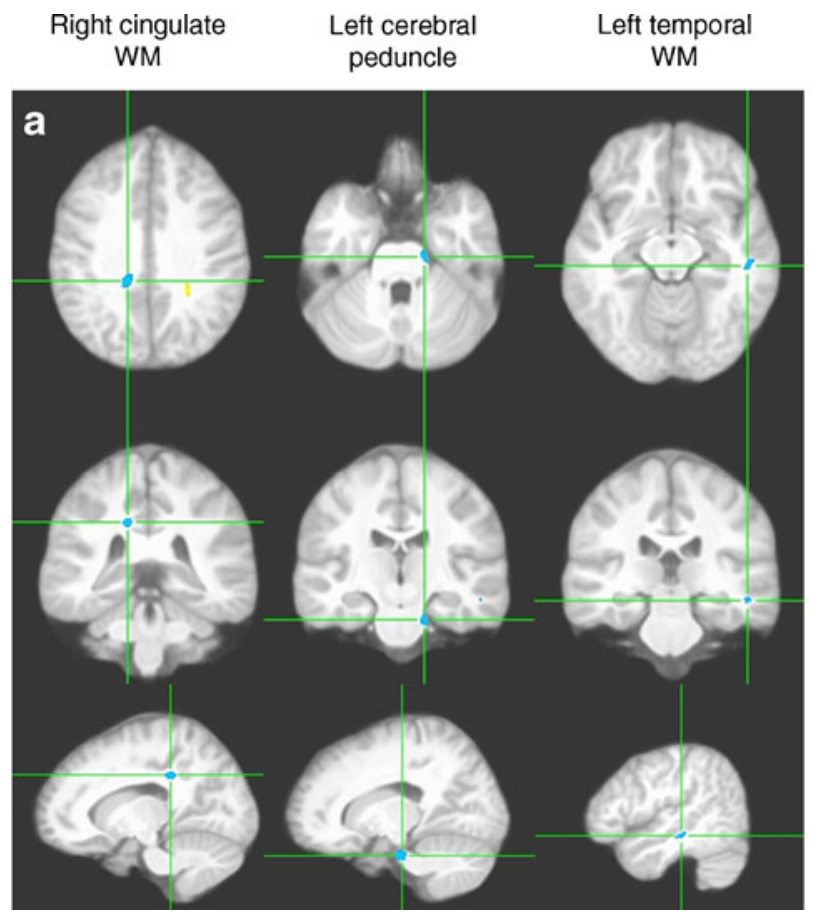

$\begin{array}{ccc}\text { Right superior } & \text { Left frontal } & \text { Right parietal } \\ \text { temporal gyrus } & \text { cortex } & \text { cortex }\end{array}$

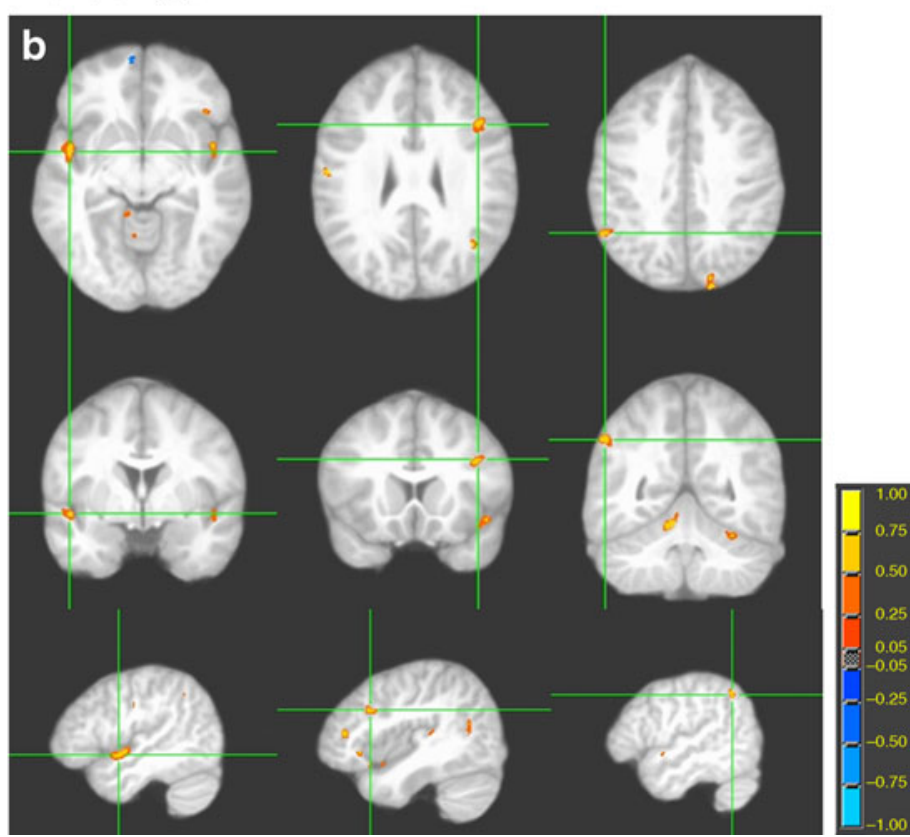

Fig. 2 Sampling of significant FA and ADC clusters. The three rows of images, each showing an orthogonal orientation (axial, coronal, and sagittal), display the clusters with the axes going through the centroid (coordinates in Table 4). (a) WM FA reductions (blue) and (b) GM ADC elevations (yellow/orange) in the type 2 diabetes group 
vascular reactivity, which is integral to well-regulated cerebral blood flow, is key for maintaining an optimal neuronal environment during brain activation [40]. Although the underlying mechanisms remain elusive, we propose that impairments in vascular reactivity, which have been shown to be present among individuals with insulin resistance or type 2 diabetes [41, 42], may contribute to the reductions in cognitive performance present among obese adolescents with type 2 diabetes. Other diabetes-associated complications, such as subclinical large vessel and microvascular abnormalities, are among possible contributing factors $[14,43]$.

Although we did not uncover CRP differences between groups-most likely because our control group was also obese-we did not evaluate inflammation in a comprehensive manner, and inflammation remains a potentially important mechanism mediating some of the adverse effects of type 2 diabetes on brain integrity [44]. Future studies should also assess body composition in more detail, as more subtle differences in adiposity may also contribute to the effects observed.

The effect sizes for the cognitive differences were larger than those reported in studies of adolescents with type 1 diabetes and adults with type 2 diabetes. Though alarming, our results are consistent with the higher complication rate in peripheral tissues in those with type 2 diabetes relative to their adolescent peers with type 1 diabetes, despite shorter disease duration [13]. Also supporting these larger effect sizes in the degree of abnormality, adolescents with type 2 diabetes have a markedly reduced life expectancy accompanied by a dramatic increase in medical complications [45]. Our diabetic participants had a mean $\mathrm{HbA}_{1 \mathrm{c}}$ of $8.3 \%$, indicating intermediate glycaemic control. Given adult data demonstrating cognitive impairments during prediabetic stages of insulin resistance [46], it is possible that subtle alterations begin during the preclinical stages, thus increasing the time of adverse exposure for these young brains. The lack of associations between ratings of obstructive sleep apnoea and cognitive performance may be explained by the fact that obesity is closely associated with sleep apnoea in children [18] and our groups were matched on both BMI and waist circumference. Future studies should measure sleep apnoea more comprehensively.

This study has two major strengths: our groups comprised adolescents with similar demographic and socioeconomic backgrounds as well as comparable BMI and waist circumference. A second strength is that our automated brain analysis methods provide unbiased, sensitive and comprehensive assessments of both gross and microstructural brain structure. This study is, however, limited by its relatively small sample size and the fact that we carried out 13 univariate comparisons for the cognitive data and six comparisons for the brain volumes, and did not correct for multiple comparisons. However, all cognitive variables were descriptively lower in the diabetic group with $6 / 13$ showing at least a statistical trend. In addition, 4/6 brainvolume variables differed significantly in the predicted direction. If we use the very conservative Bonferroni correction, the overall intellectual capacity difference and all the brain volume differences remain significant. Consequently, we consider it justified that, in this first report of brain and cognitive complications in adolescents with type 2 diabetes, we have not controlled for multiple comparisons.

This study demonstrates both brain and cognitive abnormalities among obese adolescents with type 2 diabetes. To better understand the underlying pathological mechanisms, future work should include assessments of vascular function, dynamic insulin function, lipid metabolism and inflammatory markers, as well as their interactions. Furthermore, future work should be longitudinal, thus tracking pathological changes from the preclinical stages of insulin resistance to type 2 diabetes. Given that some of our obese control participants are likely to have subtle levels of insulin resistance, it is possible that the group differences we uncovered would be even larger relative to lean adolescents. It will be essential to ascertain whether these brain abnormalities are reversible with improvements in diabetic control.

Acknowledgements The study was supported by grants from the National Institutes of Health DK070985 and supported in part by grant 1UL1RR029893 from the National Center for Research Resources and the Pollock-Nguyen Charitable Fund. None of the authors has any conflict of interest or financial disclosure to make. We thank A. Arentoft and $\mathrm{C}$. Hoogendoorn for coordinating the adolescent diabetes study and together with V. Polyakov for conducting the cognitive testing; all are affiliated with Department of Psychiatry, NYU School of Medicine, New York, NY, USA.

Duality of interest The authors declare that there is no duality of interest associated with this manuscript.

\section{References}

1. Hannon TS, Rao G, Arslanian SA (2005) Childhood obesity and type 2 diabetes mellitus. Pediatrics 116:473-480

2. Stumvoll M, Goldstein BJ, van Haeften TW (2005) Type 2 diabetes: principles of pathogenesis and therapy. Lancet 365: $1333-1346$

3. Awad N, Gagnon M, Messier C (2004) The relationship between impaired glucose tolerance, type 2 diabetes, and cognitive function. J Clin Exp Neuropsychol 26:1044-1080

4. Manschot SM, Brands AMA, van der Grond J et al (2006) Brain magnetic resonance imaging correlates of impaired cognition in patients with type 2 diabetes. Diabetes 55:1106-1113

5. Pirttila T, Jarvenpaa R, Laippala P, Frey H (1992) Brain atrophy on computerized axial tomography scans: interaction of age, diabetes and general morbidity. Gerontology 38:285-291

6. Araki Y, Nomura M, Tanaka H et al (1994) MRI of the brain in diabetes mellitus. Neuroradiology 36:101-103 
7. van Harten B, de Leeuw FE, Weinstein HC, Scheltens P, Biessels GJ (2006) Brain imaging in patients with diabetes: a systematic review. Diab Care 29:2539-2548

8. van Harten B, Oosterman J, Muslimovic D, van Loon B-JP, Scheltens P, Weinstein HC (2007) Cognitive impairment and MRI correlates in the elderly patients with type 2 diabetes mellitus. Age Ageing 36:164-170

9. Gold S, Dziobek I, Sweat V et al (2007) Hippocampal damage and memory impairments as possible early brain complications of type 2 diabetes. Diabetologia 50:711-719

10. den Heijer T, Vermeer SE, van Dijk EJ et al (2003) Type 2 diabetes and atrophy of the medial temporal lobe structures on brain MRI. Diabetologia 46:1604-1610

11. Northam EA, Rankins D, Lin A et al (2009) Central nervous system function in youth with type 1 diabetes 12 years after disease onset. Diab Care 32:445-450

12. Perantie DC, Wu J, Koller JM et al (2007) Regional brain volume differences associated with hyperglycemia and severe hypoglycemia in youth with type 1 diabetes. Diab Care 30:2331-2337

13. Eppens MC, Craig ME, Cusumano J et al (2006) Prevalence of diabetes complications in adolescents with type 2 compared with type 1 diabetes. Diab Care 29:1300-1306

14. Urbina EM, Kimball TR, McCoy CE, Khoury PR, Daniels SR, Dolan LM (2009) Youth with obesity and obesity-related type 2 diabetes mellitus demonstrate abnormalities in carotid structure and function. Circulation 119:2913-2919

15. Heilman K, Zilmer M, Zilmer K et al (2009) Arterial stiffness, carotid artery intima-media thickness and plasma myeloperoxidase level in children with type 1 diabetes. Diabetes Res Clin Pract 84:168-173

16. Mabbott DJ, Noseworthy M, Bouffet E, Laughlin S, Rockel C (2006) White matter growth as a mechanism of cognitive development in children. Neuroimage 33:936-946

17. Grieve SM, Williams LM, Paul RH, Clark CR, Gordon E (2007) Cognitive aging, executive function, and fractional anisotropy: A diffusion tensor MR imaging study. AJNR Am J Neuroradiol 28:226-235

18. Dayyat E, Kheirandish-Gozal L, Sans Capdevila O, Maarafeya MMA, Gozal D (2009) Obstructive sleep apnea in children: Relative contributions of body mass index and adenotonsillar hypertrophy. Chest 136:137-144

19. Gozal D, Kheirandish-Gozal L (2007) Neurocognitive and behavioral morbidity in children with sleep disorders. Curr Opin Pulm Med 13:505-509

20. Macey PM, Kumar R, Woo MA, Valladares EM, Yan-Go FL, Harper RM (2008) Brain structural changes in obstructive sleep apnea. Sleep 31:967-977

21. Mather KJ, Hunt AE, Steinberg HO et al (2001) Repeatability characteristics of simple indices of insulin resistance: Implications for research applications. J Clin Endocrinol Metab 86:5457-5464

22. Radziuk J (2004) Insulin sensitivity and its measurement: structural commonalities among the methods. J Clin Endocrinol Metab 85:4426-4433

23. Expert Panel (2001) Executive Summary of the Third Report of the National Cholesterol Education Program (NCEP) Expert Panel on Detection, Evaluation and Treatment of High Blood Cholesterol in Adults (Adult Treatment Panel III). JAMA 285:2486-2497

24. Mindell J, Owens J (2003) A clinical guide to pediatric sleep: diagnosis and management of sleep problems. Lippincott Williams \& Wilkins, Philadelphia

25. Lezak MD, Howieson DB, Loring DW (2004) Neuropsychological assessment. Oxford University Press, New York
26. Scheltens P, Barkhof F, Leys D et al (1993) A semiquantative rating scale for the assessment of signal hyperintensities on magnetic resonance imaging. J Neurol Sci 114:7-12

27. Basser PJ, Pierpaoli C (1996) Microstructural and physiological features of tissues elucidated by quantitative-diffusion-tensor MRI. J Magn Reson B 111:209-219

28. Irwan R, Sijens PE, Potze JH, Oudkerk M (2005) Correlation of proton MR spectroscopy and diffusion tensor imaging. Magn Reson Imaging 23:851-858

29. Good CD, Johnsrude IS, Ashburner J, Henson RNA, Friston KJ, Frackowiak RSJ (2001) A voxel-based morphometric study of ageing in 465 normal adult human brains. Neuroimage $14: 21-36$

30. Giedd JN, Blumenthal J, Jeffries NO et al (1999) Brain development during childhood and adolescence: a longitudinal MRI study. Nat Neurosci 2:861-863

31. Convit A, Wolf OT, de Leon MJ et al (2001) Volumetric analysis of the pre-frontal regions: findings in aging and schizophrenia. Psychiatry Res 107:61-73

32. Ardekani BA, Braun M, Hutton BF, Kanno I, Iida H (1995) A fully automatic multimodality image registration algorithm. J Comput Assist Tomogr 19:615-623

33. Yau PL, Javier D, Tsui WH et al (2009) Emotional and neutral declarative memory impariments and associated white matter microstructural abnormalities in adults with type 2 diabetes. Psychiatry Res 174:223-230

34. Benjamini Y, Hochberg Y (1995) Controlling the false discovery rate: A practical and powerful approach to multiple testing. J R Statist Soc B 57:289-300

35. Paus T, Collins DL, Evans AC, Leonard G, Pike B, Zijdenbos A (2001) Maturation of white matter in the human brain: a review of magnetic resonance studies. Brain Res Bull 54:255-266

36. Smith KJ, Kapoor R, Felts PA (2009) Demyelination: The role of reactive oxygen and nitrogen species. Brain Pathol 9:62-92

37. Veglio F, Paglieri C, Rabbia F, Bisbocci D, Bergui M, Cerrato P (2009) Hypertension and cerebrovascular damage. Atherosclerosis 205:331-341

38. Novak V, Last D, Alsop DC et al (2006) Cerebral blood flow velocity and periventricular white matter hyperintensities in type 2 diabetes. Diab Care 29:1529-1534

39. Benton D, Parker PY, Donohoe RT (1996) The supply of glucose to the brain and cognitive functioning. J Biol Sci 28:463-479

40. Drake CT, Iadecola C (2007) The role of neuronal signaling in controlling cerebral blood flow. Brain Lang 102:141-152

41. Stansberry KB, Shapiro SA, Hill MA, McNitt PM, Meyer MD, Vinik AI (1996) Impaired peripheral vasomotion in diabetes. Diab Care 19:715-721

42. Tooke JE, Hannemann MM (2000) Adverse endothelial function and the insulin resistance syndrome. J Intern Med 247:425-431

43. Manschot S, Biessels G, de Valk H et al (2007) Metabolic and vascular determinants of impaired cognitive performance and abnormalities on brain magnetic resonance imaging in patients with type 2 diabetes. Diabetologia 50:2388-2397

44. Hagberg H, Mallard C (2005) Effect of inflammation on central nervous system development and vulnerability. Curr Opin Neurol 18:117-123

45. Pavkov ME, Bennett PH, Knowler WC, Krakoff J, Sievers ML, Nelson RG (2006) Effect of youth-onset type 2 diabetes mellitus on incidence of end-stage renal disease and mortality in young and middle-aged Pima Indians. JAMA 296:421-426

46. Bruehl H, Sweat V, Hassenstab J, Polyakov V, Convit A (2010) Cognitive impairment in nondiabetic middle-aged and older adults is associated with insulin resistance. J Clin Exp Neuropsychol 32:487-493 